\title{
Spontaneous tumorigenicity of primary human oral keratinocytes with human papillomavirus negativity and impaired apoptosis
}

\author{
DA HYUN JANG ${ }^{1 *}$, JU-EUN OH ${ }^{1 *}$, HYUN KI KANG ${ }^{1}$, O BOK KIM ${ }^{1}$, SEUNG-KI MIN ${ }^{2}$, \\ SUNG YOUN JUNG ${ }^{1}$, SEONG-DOO HONG ${ }^{3}$, JAE-IL LEE ${ }^{3}$ and BYUNG-MOO MIN ${ }^{1}$ \\ Departments of ${ }^{1}$ Oral Biochemistry, ${ }^{2}$ Oral and Maxillofacial Surgery and ${ }^{3}$ Oral Pathology, DRI, \\ and BK21 CLS, Seoul National University School of Dentistry, Seoul 110-749, Korea
}

Received January 15, 2010; Accepted March 12, 2010

DOI: 10.3892/ijo_00000636

\begin{abstract}
Although $>60 \%$ of oral cancer cases are not related to human papillomavirus (HPV) infection, most studies of oral carcinogenesis in human cells in vitro are carried out with human oral keratinocytes immortalized by HPV DNA. To explore whether human oral keratinocytes can spontaneously transform without HPV infection, we attempted to establish spontaneously immortalized and tumorigenic-transformed human oral keratinocytes by serial subculture to the post-mitotic stage. Here we report two spontaneously transformed human oral keratinocyte lines from adult human gingival samples. These lines were obviously immortal (>140 passages) and transformed phenotypes in vitro. One of the lines, Spi-HOK1, remained nontumorigenic in nude mice, whereas the other line, Spt-HOK80, showed tumorigenicity. These lines showed epithelial originality, but did not contain high-risk types of HPV DNAs. On karyotyping, Spi-HOK1 was aneuploid with a unique stable marker chromosome. Both cell lines revealed a mutation in the $p 53$ gene, loss of $\mathrm{p} 21^{\mathrm{WAF} 1 / \mathrm{Cip} 1}$ and overexpression of $\mathrm{p}-\mathrm{Rb}$ Ser807/811. These cell lines were resistant to cisplatininduced apoptosis by suppressing induction of apoptotic proteins. These results clearly demonstrate that spontaneous immortalization and spontaneous tumorigenic transformation of primary human oral keratinocytes can occur in vitro without HPV infection and are associated with chromosomal alterations, p53 mutation and impaired apoptosis. To our knowledge, this is the first report demonstrating that the SpiHOK1 and Spt-HOK80 lines are novel cell lines that are spontaneously transformed from primary human oral keratinocytes.
\end{abstract}

Correspondence to: Dr Byung-Moo Min, Department of Oral Biochemistry, Seoul National University School of Dentistry, 28 Yeonkun-Dong, Chongno-Ku, Seoul 110-749, Korea

E-mail: bmmin@snu.ac.kr

${ }^{*}$ Contributed equally

Key words: primary human oral keratinocytes, spontaneous transformation, chromosomal alteration, p53 mutation, apoptosis

\section{Introduction}

Oral cancer is the 8th and 13th most common cancer worldwide for males and females, respectively (1). Oral squamous cell carcinoma (OSCC) is the most common malignant neoplasm of the oral cavity and accounts for $>90 \%$ of intraoral malignant tumors. Epidemiological data strongly link smoking and alcohol consumption to the development of this malignancy (2). There is also evidence that a subset of OSCCs is associated with infection by oncogenic human papillomavirus (HPV); a recent study identified HPV in $\sim 40 \%$ of cases (3). Nutritional deficiencies and dietary customs have also been implicated in the etiology of oral cancer $(4,5)$. Endogenous factors such as body heat and free radicals are also responsible for cellular DNA damage (6).

Although the etiological factors for oral cancer are wellestablished, the mechanisms through which oral cancer develops are still largely unknown, partly due to the lack of proper in vitro and in vivo models of human oral carcinogenesis. In fact, most studies of oral carcinogenesis in human cells in vitro are performed with human oral keratinocytes immortalized by HPV-16 DNA $(7,8)$; however, $>60 \%$ of OSCCs are not related to HPV infection (3). Cell lines that are representative of particular cancers make useful laboratory models to study genes and proteins implicated in the initiation and progression of cancers. Therefore, to study the multistep molecular events underlying human oral carcinogenesis, it is important to establish an in vitro multistep carcinogenesis model system using oral keratinocyte lines that are not infected with HPV.

Normal human cells will only grow for a limited number of generations in vitro before they undergo senescence, a state in which they remain metabolically active, but cease dividing (9). Serial subculture of primary normal human oral keratinocytes (NHOKs), with a limited proliferation capacity in vitro $(10,11)$, induces terminal differentiation and senescence concomitant with apoptosis (10-12). Cells from normal tissues of rodent origin can become tumorigenic when transformed in vitro by various agents and can also undergo spontaneously tumorigenic conversion after variable periods in culture (13). In contrast to rodent epidermal cells, human epidermal keratinocytes are rather resistant to transformation in vitro $(14,15)$. The differences between human and rodent cells in their sensitivity to transforming agents 
in vitro have been attributed to several discriminating speciesrelated factors, such as natural life span, degree of inbreeding and genetic stability $(16,17)$.

Since the incidence of tumorigenic transformation in different species appears to correlate with the frequency of spontaneous chromosomal aberrations, the low incidence of tumorigenic transformation of human cells in culture has been ascribed mainly to the higher stability of the human genome (16). To date, transformation of human oral keratinocytes has only been achieved reproducibly by transfection with cloned HPV-16 and -18 DNAs $(7,18)$ and simian virus 40 DNA (19), resulting in potentially immortalized but nontumorigenic cell lines. Also, the transfer of an exogenous hTERT cDNA, encoding the catalytic subunit of human telomerase, can prevent telomere shortening, overcome telomere-controlled senescence, and immortalize primary human cells, including mammary epithelial cells, keratinocytes, osteoblasts and fibroblasts (20). Although spontaneous immortalization of human epidermal keratinocytes in vitro has only rarely been observed (21-23), spontaneous immortalization and spontaneous tumorigenic transformation of human oral keratinocytes in vitro have not yet been reported.

In this study, we explored whether human oral keratinocytes can spontaneously transform without HPV infection in order to establish an in vitro multistep carcinogenesis model using HPV non-infected human oral keratinocyte lines. Further, we characterized the in vitro growth characteristics, epithelial originality, in vivo tumorigenicity, frequency of high-risk HPV infection, chromosomal composition, apoptotic ability and expression of apoptosis-related proteins.

\section{Materials and methods}

Primary cells and cell culture. Primary NHOKs were prepared and maintained as previously described (24). Briefly, NHOKs were isolated from human gingival tissue specimens from patients undergoing surgery. Oral keratinocytes were isolated from separated epithelial tissue by trypsinization, and primary cultures were established in keratinocyte growth medium containing $0.15 \mathrm{mM}$ calcium and a supplementary growth-factor bullet kit (KGM; Clonetics, San Diego, CA). Approximately $70 \%$ confluent primary human oral keratinocytes were plated at $1 \times 10^{5}$ cells per $60-\mathrm{mm}$ petri dish, and cultured until they reached $70 \%$ confluence; they were then subcultured at every $70 \%$ confluence, until they reached the post-mitotic stage of proliferation. Spontaneously transformed cells and HOK-16B, human oral keratinocytes immortalized by transfection with cloned HPV-16 genome (7), were also cultured in KGM.

Population doubling time. Growth curves were plotted over a 6-day period. Cells were plated at $2 \times 10^{4}$ cells $/ 9.5-\mathrm{cm}^{2}$ in a culture plate. Every $24 \mathrm{~h}$, three replicative plates were trypsinized, and viable cells were counted with a hemocytometer. After establishing the growth curves, the population doubling (PD) times of the established cell lines were estimated from the exponential growth phase.

Immunoperoxidase staining for keratin. Monolayer cells grown on glass slides were fixed in ice-cold ethanol for $10 \mathrm{~min}$, air dried and stored in a sealed box at $-20^{\circ} \mathrm{C}$ until used. Slides were rehydrated, treated with $3 \%$ hydrogen peroxide, and stained with pan-specific monoclonal mouse anti-keratin (AE1 \& AE3; Dako Corp., Santa Barbara, CA) according to the manufacturer's recommendations. Keratin was detected using the peroxidase-anti-peroxidase method with aminoethyl-carbazole as the chromogen and Harris' hematoxylin as the counterstain. Monolayer cells were also stained with normal rabbit serum as negative controls.

Determination of HPV infection. The presence of high-risk HPV (type 16, 18 and 33) DNA was determined by using the Takara PCR human papillomavirus detection set (Takara, Otsu, Japan). The primers amplified the E6 gene of the three types of HPV. The viral gene was amplified with DNA isolated from cells using the conditions recommended by the manufacturer.

In vivo tumorigenicity test. The in vivo tumorigenicity of cells was assessed in nude mice. All experimental procedures were approved by the Experimental Animal Committee of Seoul National University. Monolayer cultures were trypsinized and resuspended in culture medium. We then injected $0.3 \mathrm{ml}$ of phosphate-buffered saline containing $5 \times 10^{6}$ cells subcutaneously into athymic nude mice (BALB/cByJ-Hfhl $1^{\text {nu}}$; Orient BIO Inc., Seongnam, Korea). All mice received injections in the right flank and were monitored twice weekly for the appearance of tumors over a period of three months.

Cytogenetic analysis. Cells were treated with uridine $(0.4 \mathrm{mM})$ and fluorodeoxyuridine $(5 \mu \mathrm{M})$ for $16 \mathrm{~h}$ at $37^{\circ} \mathrm{C}$, followed by colcemid $(10 \mu \mathrm{g} / \mathrm{ml})$ for $5 \mathrm{~h}$ at $37^{\circ} \mathrm{C}$. The adherent cells were removed with a cell scraper and treated with hypotonic $\mathrm{KCl}$ $(0.075 \mathrm{M})$ for $30 \mathrm{~min}$ followed by fixation in fresh methanol: glacial acetic acid $(3: 1, \mathrm{v} / \mathrm{v})$ for $10 \mathrm{~min}$ at room temperature. Slides were stained by the trypsin-Giemsa technique, and at least 20 metaphases were evaluated for each cell line.

Cell cycle distribution analysis by flow cytometry. Cell cycle distributions were measured by fluorescence-activated cell sorting (FACS) analysis. Cells were plated at a density of $5 \times 10^{6}$ cells $/ 100-\mathrm{mm}$ dishes, adapted for $24 \mathrm{~h}$, incubated with/without $25 \mu \mathrm{M}$ cisplatin for $24 \mathrm{~h}$ at $37^{\circ} \mathrm{C}$, and detached by trypsinization. Cells were collected, washed once with PBS and fixed in $70 \%$ ethanol at $-20^{\circ} \mathrm{C}$ for at least $30 \mathrm{~min}$. Cells were washed twice with PBS and incubated for $30 \mathrm{~min}$ at $37^{\circ} \mathrm{C}$ in PBS containing $100 \mu \mathrm{g}$ RNase A and $10 \mu \mathrm{g}$ propidium iodide. DNA content and cell-cycle analysis were assessed using FACScalibur (BD Biosciences, San Jose, CA).

DNA sequencing analysis. Randomly primed cDNAs were reverse-transcribed from $5 \mu \mathrm{g}$ of total RNA using Superscriptase (Gibco BRL, Grand Island, NY, USA) in $40 \mu 1$ of mixture. cDNA mixture $(2 \mu 1)$ was used to amplify the p53 transcript (exon 1-11). PCR products were cloned into pCRII using a TA ${ }^{\mathrm{TM}}$ Cloning Kit (Invitrogen, San Diego, CA) under the conditions recommended by the manufacturer. The nucleotide sequence of the cloned DNA was determined by a Taq dideoxy terminator cyclic sequencing kit on an ABI 
377 automatic DNA sequencer (Perkin-Elmer, Foster City, CA).

Western blot analysis. Western blot analysis was performed as in previous reports (24) using antibodies to p53 (PAb 1801), (Oncogene Sciences, Uniondale, NY), p16 (C-20), p21 WAF1/Cip1 (187) (Santa Cruz Biotechnology, Santa Cruz, CA), p-Rb (Ser780; no. 9307), p-Rb (Ser807/811; no. 9308), Rb (4H1; no. 9309), caspase-3 (no. 9662), caspase-8 (no. 9746), caspase-9 (no. 9502), Bax (no. 2774) and poly (ADP-ribose) polymerase (PARP; no. 9542) (Cell Signaling Technology, Danvers, MA). After probing with each antibody, the membrane was subjected to immunoblot analysis using a specific antibody to $\beta$-actin for determination of the total protein loaded per lane.

RT-PCR. Involucrin mRNA levels were determined by reverse transcription-polymerase chain reaction (RT-PCR). Total RNA was reverse transcribed to $\mathrm{CDNA}$, which was used for PCR amplification of involucrin (40 cycles) and GAPDH (30 cycles, denaturation at $95^{\circ} \mathrm{C}$ for $20 \mathrm{sec}$, annealing at $60^{\circ} \mathrm{C}$ for $10 \mathrm{sec}$, and extension at $70^{\circ} \mathrm{C}$ for $10 \mathrm{sec}$ ). Involucrin primers were designed as follows: forward, 5'-GGCCACCCAAACATAA ATAACCAC-3'; reverse, 5'-CACCTAGCGGACCCGAAAT AAGT-3'. GAPDH primers for normalization of the expression level were also prepared: 5'-CCATCTTCCAGGAGCG AGATC-3' and 5'-GCCTTCTCCATGGTGGTGAA-3'. The PCR products were resolved on a $1.5 \%$ agarose gel, stained with ethidium bromide, and visualized using a UV illuminator.

Real-time RT-PCR. Total RNA was prepared from the cells using TRI reagent (Molecular Research Center, Cincinnati, $\mathrm{OH})$. The cDNA was prepared using SuperScript ${ }^{\mathrm{TM}}$ III reverse transcriptase (Invitrogen) and a random hexamer and then subjected to real-time PCR amplification using SYBRGreen PCR master mix (Takara). The reaction contained a $300 \mathrm{nM}$ final concentration of each primer and cDNA, corresponding to $50 \mathrm{ng}$ of total RNA. After incubation at $95^{\circ} \mathrm{C}$ for $4 \mathrm{~min}$, samples were subjected to 40 cycles of amplification at $95^{\circ} \mathrm{C}$ for $15 \mathrm{sec}$ followed by $60^{\circ} \mathrm{C}$ for $20 \mathrm{sec}$ and $72^{\circ} \mathrm{C}$ for $31 \mathrm{sec}$. Each assay was normalized to HPRT1 mRNA. The normalized data were expressed as the fold increase against the mRNA level of NHOKs. The real-time PCR primers used were as follows: p53, 5'-CTTGCCGTCC CAAGCAAT-3' (sense) and 5'-TGGCATTCTGGGAGCTT CAT-3' (antisense); p21, 5'-CTGGAGACTCTCAGGGT CGAA-3' (sense) and 5'-GCGGATTAGGGCTTCCTCTT-3' (antisense); $H P V-16$ E6, 5'-TGCACAGAGCTGCAAACA ACT-3' (sense) and 5'-CCCGAAAAGCAAAGTCATATA CCT-3' (antisense); HPV-18 E6, 5'-TACCTGATCTGTGCA CGGAACT-3' (sense) and 5'-ACCGCAGGCACCTCTGT AAG-3' (antisense); HPRT1, 5'-GGTCAGGCAGTATAAT CCAAAGA-3' (sense) and 5'-GGGCATATCCTACAACA AACT-3' (antisense).

Statistical analysis. All experiments were performed at least in triplicate. Data are expressed as mean \pm SD. The statistical significance of results was analyzed with one-way ANOVA followed by Scheffe's adjustment or Student's t-test. Difference were considered significant at $\mathrm{P}<0.05$.

\section{Results}

Two spontaneously transformed oral keratinocyte lines isolated from human gingival specimens. Oral keratinocytes from full thickness adult human gingival specimens were isolated by separation of the epidermis and dermis with trypsin at $37^{\circ} \mathrm{C}$ and plated at high cell density. Since longterm growth in primary human oral keratinocytes was optimal in low $\mathrm{Ca}^{2+}(0.2 \mathrm{mM})$, probably due to a marked reduction in terminal differentiation $(7,21)$, primary cultures were established in KGM containing $0.15 \mathrm{mM}$ calcium and a supplementary growth factor bullet kit. Primary cultures were then subcultured at every $70 \%$ confluence until they reached the post-mitotic stage of proliferation.

Primary NHOKs and lower passages of the cells displayed typical keratinocyte morphology and retained an undifferentiated phenotype with distinct intercellular spaces (Fig. 1Aa). Serial subculture of primary NHOKs to the post-mitotic stage (passages 6-10 depending on donor age), however, induced terminal differentiation, with highly enlarged and elongated cell bodies and terminally differentiated cells rounded up and became detached from the culture dish in a time-dependent manner (data not shown). These results are consistent with those from our previous report (10) and indicate that subculture of primary NHOKs results in terminal differentiation, leading to cell death. In contrast, among $>350$ primary cultures, two individual cultures continued to proliferate beyond terminal differentiation, although most cells became detached from the culture dish. In these cases, male gingival specimens were obtained from the periphery of mandibular premolars. By passage 8 , small keratinocytes appeared in the cultures and proliferated well, and no large, terminally differentiated keratinocytes were evident in these cells. The resulting population of small keratinocytes that survived this putative crisis period appeared morphologically uniform and produced colonies of keratinocytes exhibiting typical keratinocyte characteristics, including cell-cell adhesion (data not shown). The keratinocytes continued to proliferate and could subsequently be passaged by trypsinization (split ratio 1:2). Subculturing with routine methods (EDTA/trypsin) was successful; the cells were reattached after transfer to a new dish and grew, and cell density was not critical to maintenance of satisfactory proliferation. Keratinocyte growth was maintained through at least 140 passages, demonstrating the cells had achieved immortality. During propagation for $>140$ passages (undergoing at least 260 PDs), no obvious crises were noted. One resulting cell line was designated as SpiHOK1 and the other as Spt-HOK80 to denote their origins from human adult oral keratinocytes.

Spontaneously immortalized Spi-HOK1 and spontaneously tumorigenic-transformed Spt-HOK80 lines display keratinocyte characteristics. Spi-HOK1 remained predominantly as a monolayer, but Spt-HOK80 cells grew stratiform. Both cell lines displayed squamous cell-shape morphology, although some cells were spindle-shaped or polyhedral (Fig. 1A). The PD times of the Spi-HOK1 and Spt-HOK80 in KGM were approximately 30 and $29 \mathrm{~h}$, respectively (Fig. 1B), which is significantly shorter than that of HOK-16B (47 h). These cells also expressed keratin, the marker for epithelial cells, 
A

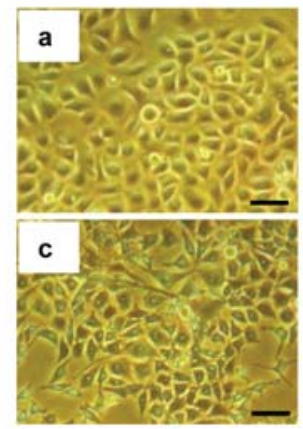

B
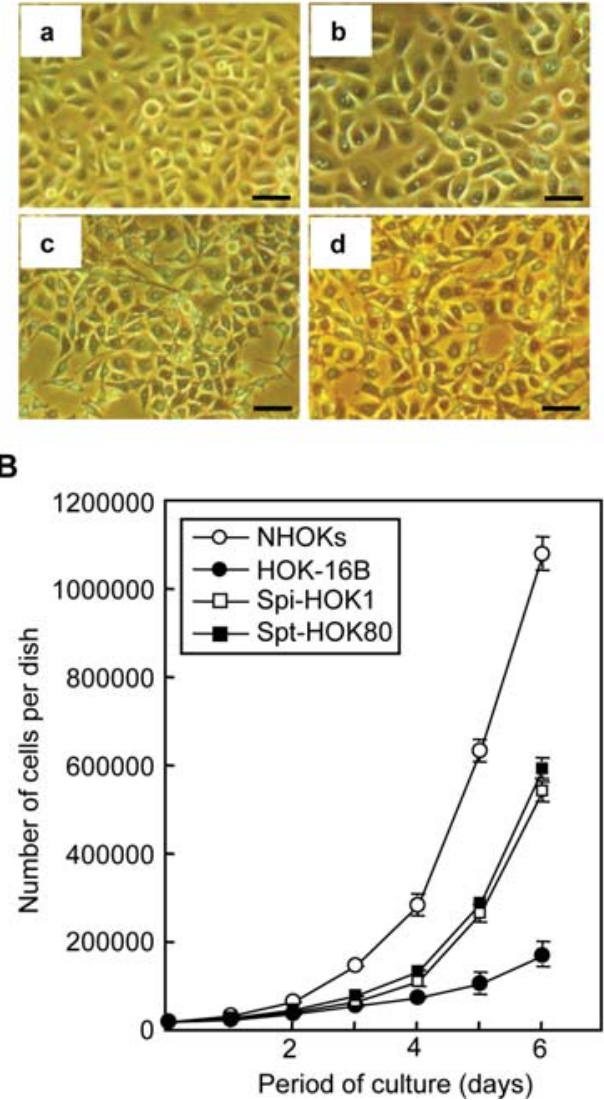

C
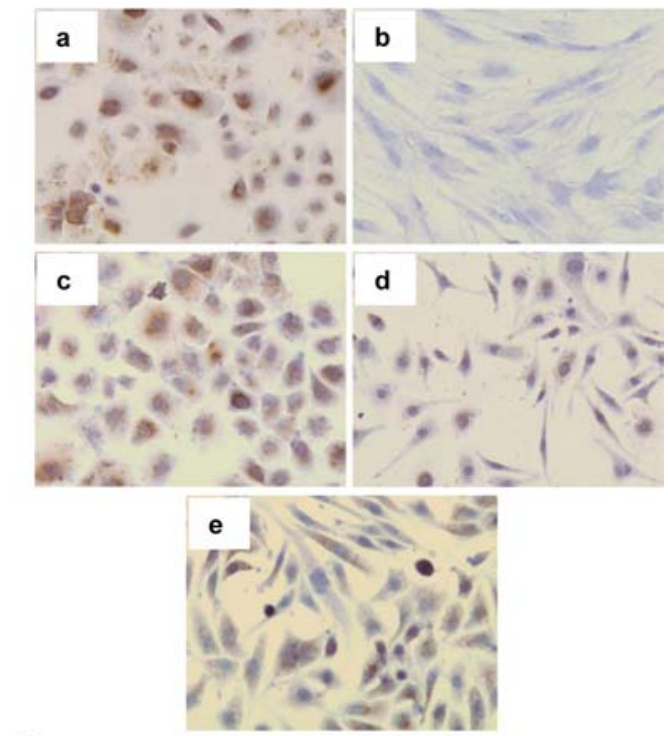

D

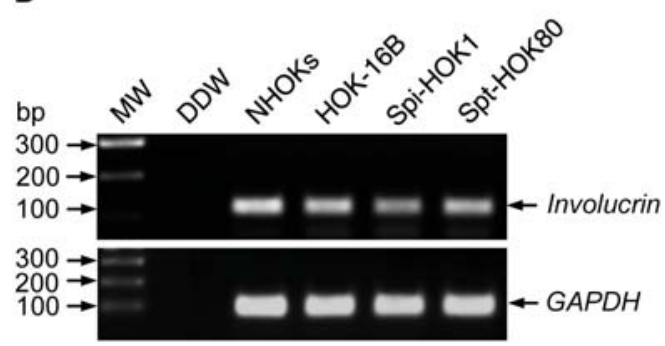

Figure 1. Morphological features and general characteristics of spontaneously transformed Spi-HOK1 and Spt-HOK80 lines. Two spontaneously transformed oral keratinocyte lines were established from adult human gingival samples by serial subculture to the post-mitotic stage. Microscopic features (A) and growth curves (B) of NHOKs (a, PD 12.2), HOK-16B (b, passage 61) and two spontaneously transformed human oral keratinocytes Spi-HOK1 (c, passage 78) and Spt-HOK80 (d, passage 74). Scale bars: $50 \mu \mathrm{m}$. (C) Immunoperoxidase staining for keratins. a, NHOKs; b, normal human gingival fibroblasts; c, HOK-16B; d, Spi-HOK1; e, Spt-HOK80. (D) Involucrin mRNA expression detected by RT-PCR analysis. NHOKs (passage 2) were used as a positive control. GAPDH was used as an internal control.

A

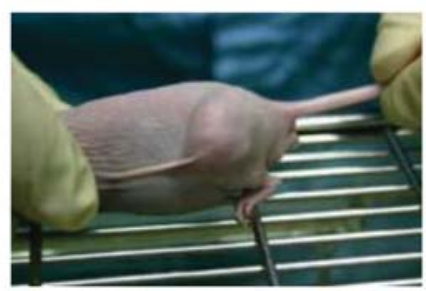

C

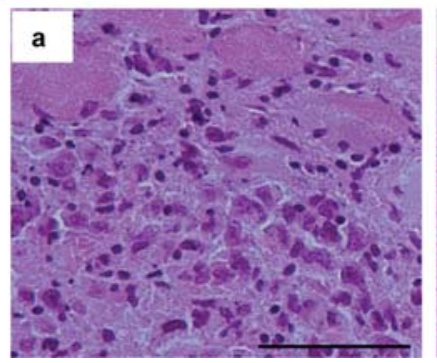

B

\begin{tabular}{lc}
\hline Cells & $\begin{array}{c}\text { Tumorigenicity in nude mice } \\
\text { (no. of mice with tumor/ } \\
\text { no of mice tested) }\end{array}$ \\
\hline NHOKs & $0 / 5$ \\
Spi-HOK1 & $0 / 10$ \\
Spi-HOK80 & $8 / 10$ \\
\hline
\end{tabular}

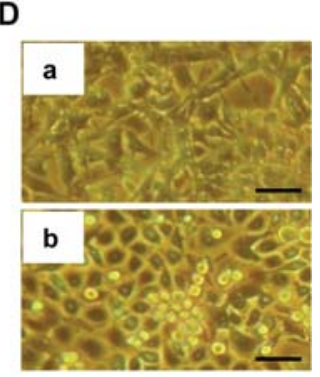

Figure 2. In vivo tumorigenicity of the spontaneously transformed Spi-HOK1 and Spt-HOK80 lines. (A) A tumor developed subcutaneously in a nude mouse by Spt-HOK80 cells. (B) Tumor incidence in nude mice receiving NHOKs, Spi-HOK1 and Spt-HOK80 cells. Monolayer cultures were trypsinized and resuspended in culture medium. PBS $(0.3 \mathrm{ml})$ containing $5 \times 10^{6}$ cells was then injected subcutaneously into athymic nude mice $\left(\mathrm{BALB} / \mathrm{cByJ}-H \mathrm{fh} 11^{\mathrm{nu}}\right)$. All mice received injections in the right flank and were monitored twice weekly for the appearance of tumors over a period of three months. (C) Microscopically, the tumors that developed from injection of Spt-HOK80 cells had a poorly differentiated squamous cell carcinoma histology (a). Infiltration of tumor cells into mouse skeletal muscle (b). Scale bars: $100 \mu \mathrm{m}$. (D) Microscopic features of Spt-HOK80-T cells, which were established from an original Spt-HOK80 tumor developing in a nude mouse, grown in KGM (a, passage 37) and DMEM (b, passage 37). Scale bars: $50 \mu \mathrm{m}$. 
based on immunohistochemical analysis (Fig. 1C). To further confirm that the transformed cells were epithelial cells, expression of involucrin, one of the molecular markers for keratinocyte differentiation (12), was examined by RT-PCR analysis. Spi-HOK1 and Spt-HOK80 expressed involucrin mRNA, although the expression levels varied between the cell lines (Fig. 1D). These results indicate that both SpiHOK1 and Spt-HOK80 originated from epithelial cells.

Because the Spi-HOK1 and Spt-HOK80 cell lines are known to be immortalized, an in vivo tumorigenicity test was performed to test whether Spi-HOK1 and Spt-HOK80 are tumorigenic-transformed. An in vivo tumorigenicity test showed that neither NHOKs nor Spi-HOK1 (passage 40) produced tumors in nude mice within three months of injection (Fig. 2B). Spt-HOK80 (passage 40) however, produced tumors in nude mice; $80 \%$ of nude mice injected with the Spt-HOK80 developed tumors at the injection site (Fig. 2A and B). Tumors began to appear four weeks after injection, followed by steady growth. Microscopically, the tumors exhibited a poorly differentiated squamous cell carcinoma histology (Fig. 2Ca) and infiltration into skeletal muscle tissue (Fig. 2Cb). Fig. 2D shows the morphology of tumorigenic cells, Spt-HOK80-T, which were established from an original Spt-HOK80 tumor developing in a nude mouse, and the cells grew stratiform in both KGM (Fig. 2Da) and DMEM (Fig. 2Db). The PD time of the Spt-HOK80-T in KGM was approximately $34 \mathrm{~h}$, which is longer than that of the Spt-HOK80 (29 h). Similar to the Spt-HOK80 cells, the Spt-HOK80-T cells proliferated well in DMEM supplemented with $10 \%$ FBS, a medium that contains a physiological level of calcium $(1.5 \mathrm{mM})$ and lesser amounts of growth factors compared to the KGM (Fig. 2Db). The PD times of the Spt-HOK80 and Spt-HOK80-T cells in DMEM were approximately 26 and $28 \mathrm{~h}$, respectively. These results indicate that Spi-HOK1 and Spt-HOK80 are spontaneously immortalized and spontaneously tumorigenic-transformed cell lines, respectively.

Spontaneously transformed Spi-HOK1 and Spt-HOK8O lines are not infected with high-risk HPVs. Because high-risk HPVs have been found in OSCCs and a large number of studies have examined the relationship between HPV infection and squamous cell carcinoma of the head and neck region, HPV infection (type 16, 18 and 33) in the Spi-HOK 1 and SptHOK80 cell lines was evaluated using PCR. The HOK-16B cells contained HPV-16 DNA (Fig. 3A). This agrees with results from our previous study showing that the HOK-16B line, an immortalized primary human oral keratinocytes by transfection with cloned HPV-16 DNA, contains 25 copies of intact HPV-16 DNA as an integrated form per cell and expresses several viral RNAs (7). The Spi-HOK1 and SptHOK80 lines, however, proved to be free of HPV DNA (Fig. 3A). Thus, to confirm that the spontaneously transformed cells are not infected with high-risk HPVs, we investigated whether high-risk HPVs are expressed in Spi-HOK1 and Spt-HOK80. Real-time RT-PCR analysis showed that the high-risk $H P V$ (type 16 and 18) E6 genes were not expressed in the Spi-HOK1 and Spt-HOK80 cell lines (Fig. 3B). Overexpression of $\mathrm{p} 16^{\mathrm{INK} 4 \mathrm{~A}}$ is highly correlated with the presence of HPV in cervical and in head and neck carcinomas (25-28)
A

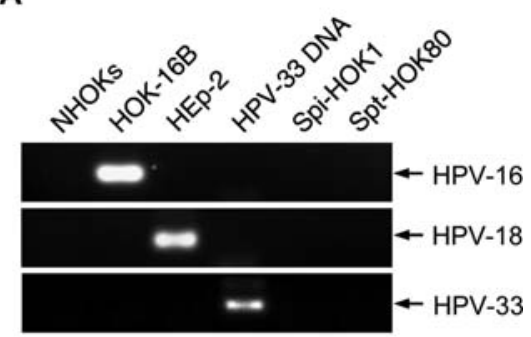

B
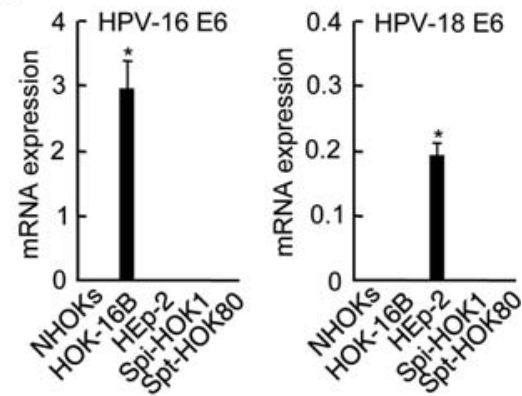

C

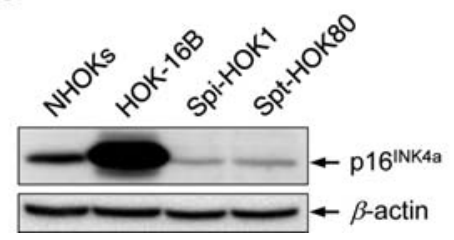

Figure 3. Determination of HPV infection and the typing of HPV by typespecific PCR analysis. (A) NHOKs were used as a negative control for HPV infection. HOK-16B and HEp-2 were used as positive controls for HPV-16 and HPV-18 infections, respectively. HPV-33 control DNA was used as a positive control for HPV-33 infection. E6 genes from HPV-33 control DNA and HPV-16- and -18-infected cells were amplified as 70 and $141 \mathrm{bp}$, respectively. (B) Quantitative real-time PCR analysis of the expression of HPV-16 E6 and HPV-18 E6 genes in the Spi-HOK1 and Spt-HOK80 lines. HPRT1 was included as an internal control. The data were normalized relative to HPRT1 amplification. Data are expressed as the mean $\pm \mathrm{SD}$ of three independent experiments. Significant difference versus NHOKs; ${ }^{*} \mathrm{P}<0.05$. (C) Western blot analysis of $\mathrm{p} 16^{\mathrm{INK} 4 \mathrm{~A}}$, a surrogate marker for HPV infection, in two spontaneously transformed cell lines Spi-HOK1 and SptHOK80. NHOKs and HOK-16B were used as negative and positive controls for HPV infection, respectively.

and has been used as a surrogate marker for HPV (25). Therefore, to diminish the possibility that other types of HPV could have infected spontaneously transformed oral keratinocytes, we further investigated the expression levels of $\mathrm{p} 16^{\mathrm{INK} 4 \mathrm{~A}}$ in the Spi-HOK1 and Spt-HOK80 cell lines using Western blot analysis. As expected, the HOK-16B cells, which contained HPV-16 DNA showed overexpression of p16 $6^{\mathrm{INK} 4 \mathrm{~A}}$; however, the expression levels of p16 $6^{\mathrm{INK} 4 \mathrm{~A}}$ in the Spi-HOK1 and SptHOK80 cell lines was notably lower than that of either NHOKs or HPV-16-immortalized HOK-16B cells (Fig. 3C). Taken together, these results suggest that spontaneously transformed Spi-HOK1 and Spt-HOK80 lines are not associated with HPV infection and that spontaneous transformation of human oral keratinocytes can occur in vitro without HPV infection.

Spontaneously transformed Spi-HOK1 and Spt-HOK8O lines exhibit aneuploidy and structural aberrations. Chromosomal analyses of Spi-HOK1 and Spt-HOK80 revealed human male 
Table I. The composite karyotype of the Spi-HOK1 and Spt-HOK80 lines.

Cell line Karyotype

Spi-HOK1

$57, \mathrm{XXY}, \operatorname{del}(1)(\mathrm{p} 32),+\mathrm{i}(1)(\mathrm{p} 10),+3,+5,+6,+\mathrm{i}(7)(\mathrm{p} 10), \operatorname{der}(8) \mathrm{i}(8)(\mathrm{q} 10),+\mathrm{i}(8)(\mathrm{p} 10),+\operatorname{del}(9)(\mathrm{q} 13)$, $+17,+21,+\operatorname{del}(22)(\mathrm{q} 11.2)[12] / 58, \mathrm{XXY}, \operatorname{del}(1)(\mathrm{p} 32),+\mathrm{i}(1)(\mathrm{p} 10),+3,+5,+6,+\mathrm{i}(7)(\mathrm{p} 10), \operatorname{der}(8) \mathrm{i}(8)$ $(\mathrm{q} 10),+\mathrm{i}(8)(\mathrm{p} 10),+\operatorname{del}(9)(\mathrm{q} 13),+17,+21,+\operatorname{del}(22)(\mathrm{q} 11.2),+\operatorname{mar}[8]$

Spt-HOK80

$45-70, \mathrm{XXY}, \operatorname{del}(1)(\mathrm{p} 32),+\mathrm{i}(1)(\mathrm{p} 10),+3,+5,+6,+\mathrm{i}(7)(\mathrm{p} 10), \operatorname{der}(8) \mathrm{i}(8)(\mathrm{q} 10),+\mathrm{i}(8)(\mathrm{p} 10),+\operatorname{del}(9)$

$(\mathrm{q} 13),+17,+21,+\operatorname{del}(22)(\mathrm{q} 11.2)[\mathrm{cp} 20]$

\section{A}

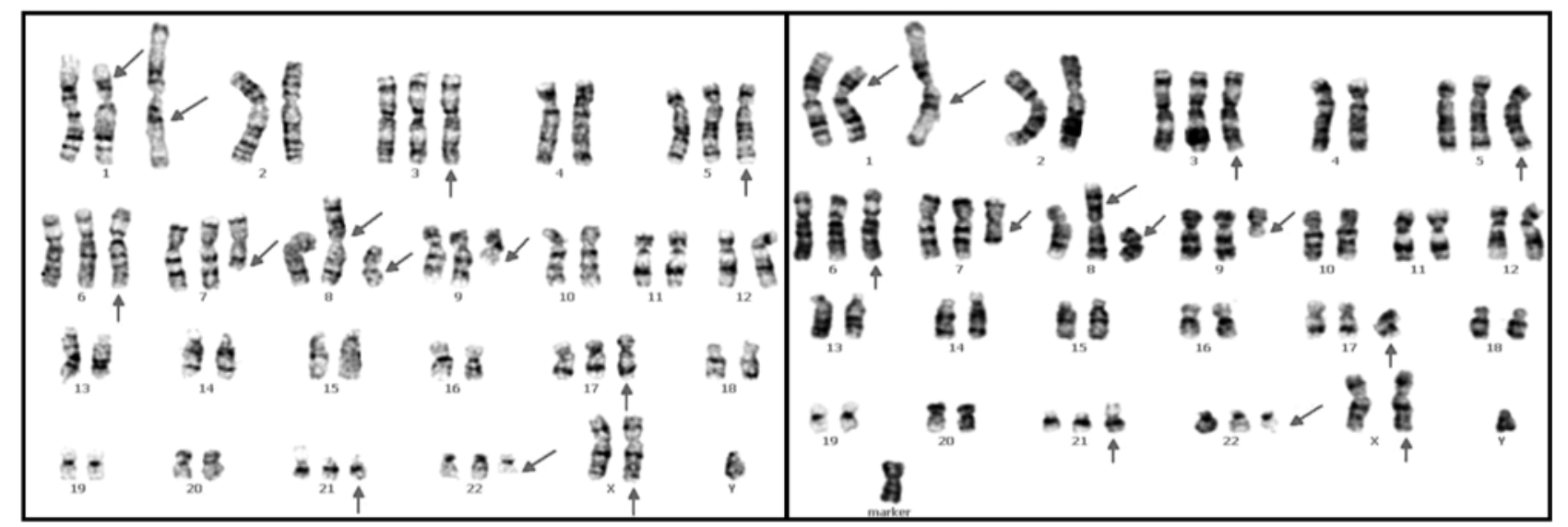

B

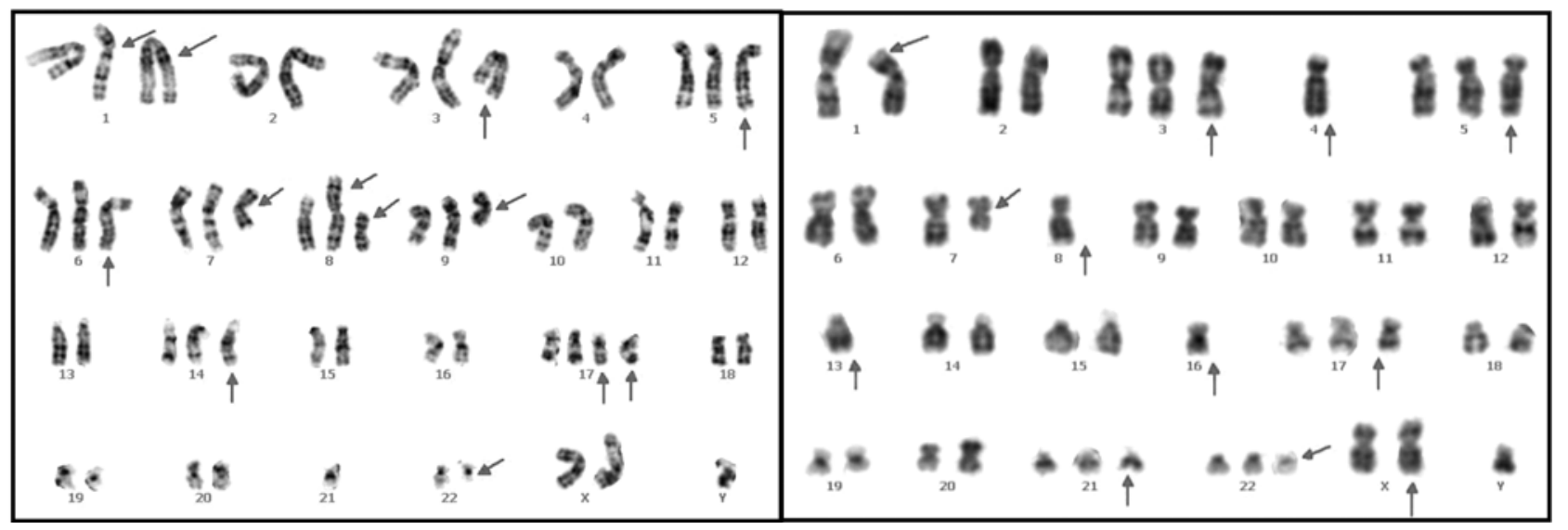

Figure 4. Karyotype analyses of the two spontaneously transformed cell lines. Karyotype analyses of Spi-HOK1 (A, passage 75) and Spt-HOK80 (B, passage 35) showing a male karyotype with random gains and losses of regions and whole chromosomes. The arrows point to the structural karyotypic alterations $(\swarrow)$ and trisomies in chromosome $(\uparrow)$.

karyotypes. Distinct numerical and structural karyotypic alterations were obvious (Fig. 4). Spi-HOK1 had 57 chromosomes resulting from full or partial trisomies of chromosomes. One marker chromosome was present in $40 \%$ of the metaphases of Spi-HOK1 (Fig. 4A and Table I). Additionally, structural aberrations were found in $100 \%$ of metaphases. Spt-HOK 80 had an abnormal number of chromosomes ranging between 45 and 70 with random gains or losses of entire chromosomes or regions of the chromosomes (Fig. 4B and Table I). The composite karyotype of each cell line is detailed in Table I.
Spontaneously transformed Spi-HOK1 and Spt-HOK80 lines display inactivation of $p 53$ and overexpression of $p-R b$. Although the mechanism of spontaneous tumorigenicity of oral keratinocytes is unclear, substantial available evidence indicates that the inactivation of tumor suppressor genes underlies cancer development. Among these, mutations in p53 are the most common genetic alteration in a variety of human tumors including OSCCs and cell lines $(29,30)$. Cells with inactivated p53 may gain a selective growth advantage, which may lead to tumor progression. In this study, Spi- 
A

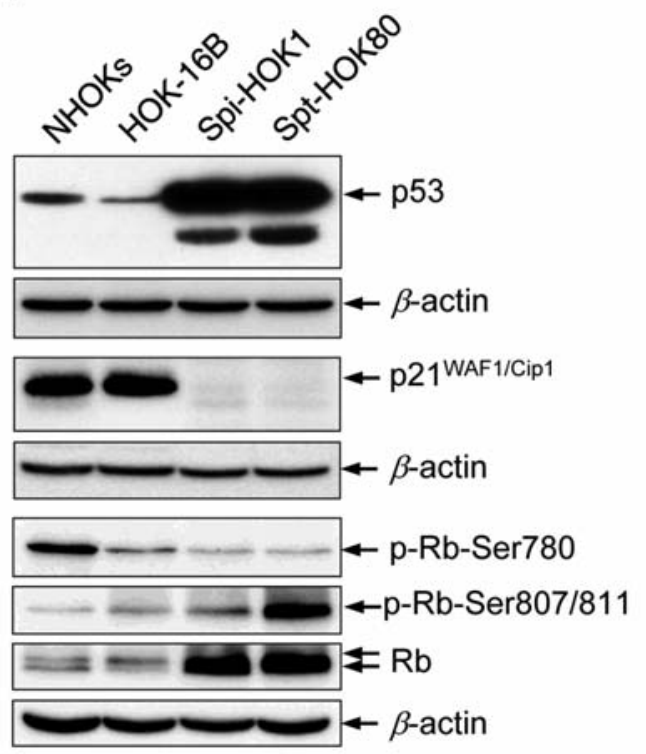

B

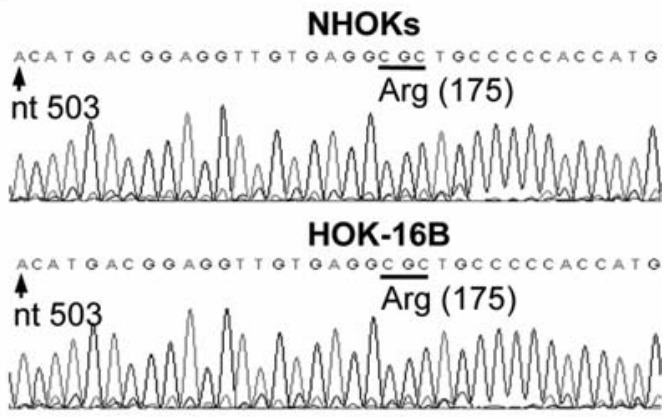

Spi-HOK1

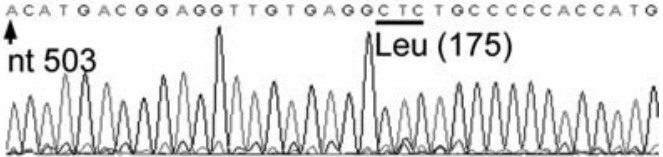

Spt-HOK80

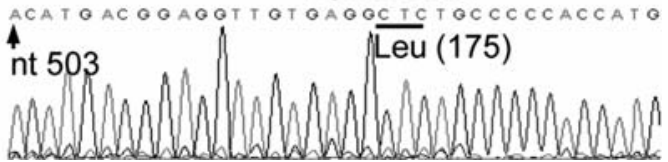

C
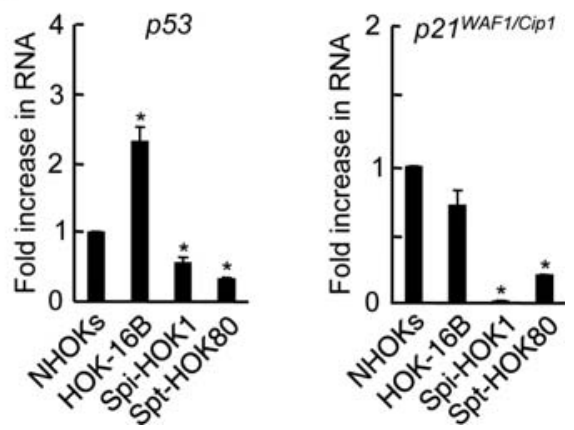

Figure 5. Biochemical analysis of the two spontaneously transformed cell lines. (A) Western blot analysis of tumor suppressor proteins. (B) Nucleotide sequencing analysis of $p 53$ cDNAs in NHOKs, HOK-16B, Spi-HOK1 and Spt-HOK80. The sequences were read in the antisense direction. (C) Quantitative real-time PCR analysis of the expression of $p 53$ and $p 21^{\text {WAFl/Cipl }}$ genes in NHOKs, HOK-16B, Spi-HOK1 and Spt-HOK80. Assay conditions were the same as described in the legend to Fig. 3B. Data are expressed as the mean $\pm \mathrm{SD}$ of three independent experiments. Significant difference versus $\mathrm{NHOKs} ;{ }^{*} \mathrm{P}<0.05$.
HOK1 and Spt-HOK80 lines contained approximately 35-40 times higher levels of p53 protein compared to NHOKs when determined by Western blot analysis (Fig. 5A). In contrast, the HOK-16B line had notably lower levels of the wild-type p53 protein, and its level was approximately one third of that found in NHOKs. This result is consistent with that of our previous reports $(8,31)$. Most point mutations in $p 53$ extend the half-life of the $\mathrm{p} 53$ protein, resulting in a higher level of p53 protein in cells (32). Thus, the presence of mutations in p53 was examined by performing DNA sequencing of whole exons (exon 1-11) of the $p 53$ gene in the NHOKs, HOK-16B, Spi-HOK1 and Spt-HOK80 cells. As expected, the spontaneously transformed Spi-HOK1 and Spt-HOK80 lines had a mutation in the $p 53$ gene. These cell lines had a mutation of CGC to $\mathrm{CTC}(\mathrm{G} \rightarrow \mathrm{T}$ transversion) at codon 175 resulting in the substitution of arginine with leucine (Fig. 5B). The sequences were confirmed using at least four independent clones. No $p 53$ mutations were found in the amplified region of $p 53$ cDNA originating from either the NHOKs or HOK16B line (Fig. 5B). The expression of $p 53$ was further examined by real-time RT-PCR analysis, and the expression levels were maintained at low levels in the Spi-HOK1 and Spt-HOK80 lines compared to those of NHOKs and HOK16B (Fig. 5C). Since $p 21^{\text {WAFI/Cipl }}$ can be directly regulated by p53 protein and can itself suppress tumor cell growth in culture (33), we analyzed the effect of $p 53$ mutation on the expression of $\mathrm{p} 21^{\mathrm{WAF} 1 / \mathrm{Cip} 1}$ by Western blot analysis. p21 ${ }^{\mathrm{WAF} 1 / \mathrm{Cip} 1}$ protein was not detected in the Spi-HOK1 and Spt-HOK80 lines (Fig. 5A). The expression of $p 21^{\text {WAFl/Cipl }}$ was further examined by real-time RT-PCR analysis, and the expression levels were maintained at marginal levels in the Spi-HOK1 and Spt-HOK80 lines (Fig. 5C), suggesting that the expression of $\mathrm{p} 21^{\mathrm{WAF} 1 / \mathrm{Cip} 1}$ is regulated at the transcriptional level. Since $\mathrm{Rb}$ protein is a DNA-binding phosphoprotein that plays a key role in the regulation of cellular proliferation, we further investigated $\mathrm{p}-\mathrm{Rb}$ levels from the NHOKs, HOK16B, Spi-HOK1 and Spt-HOK80 cells. All cells expressed $\mathrm{Rb}, \mathrm{p}-\mathrm{Rb}$-Ser780 and p-Rb-Ser807/811 proteins. In particular, the levels of p-Rb-Ser807/811 were steadily increased during the process of increased tumorigenicity of human oral keratinocytes. Similarly, the levels of Rb in Spi-HOK1 and Spt-HOK80 lines were markedly higher than that of NHOKs. In contrast, the levels of p-Rb-Ser780 were decreased during the process of increased tumorigenicity (Fig. 5A). These results strongly suggest that inactivation of tumor suppressor p53 by point mutation and overexpression of p-Rb-Ser807/ 811 are associated with spontaneous tumorigenicity of human oral keratinocytes.

Spontaneously transformed Spi-HOK1 and Spt-HOK8O lines show decreased sensitivity to cisplatin-induced apoptosis by suppressing induction of apoptotic proteins. As impaired apoptosis plays a central role in the pathogenesis of many diseases, including tumorigenesis (34), we investigated the apoptotic ability of cells. When NHOKs, Spi-HOK1 and SptHOK80 cells were cultured for $24 \mathrm{~h}$ in the presence of $25 \mu \mathrm{M}$ cisplatin, cell death, which was analyzed by flow cytometry, was induced (Fig. 6A). Cisplatin treatment in NHOKs, SpiHOK1 and Spt-HOK80 cells induced a significant increase in the sub-G1 fraction, which is indicative of cell apoptosis; 
A
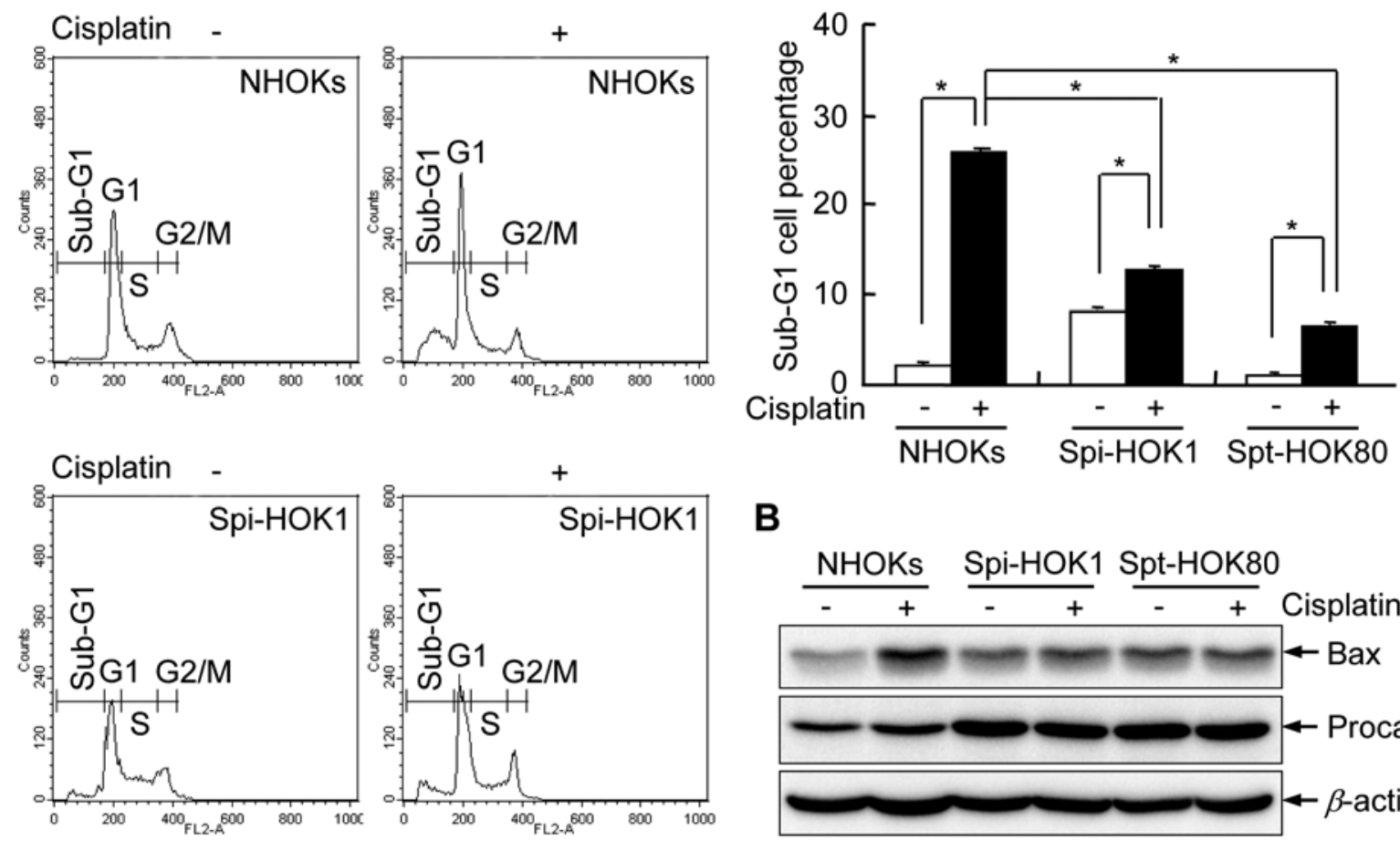

B
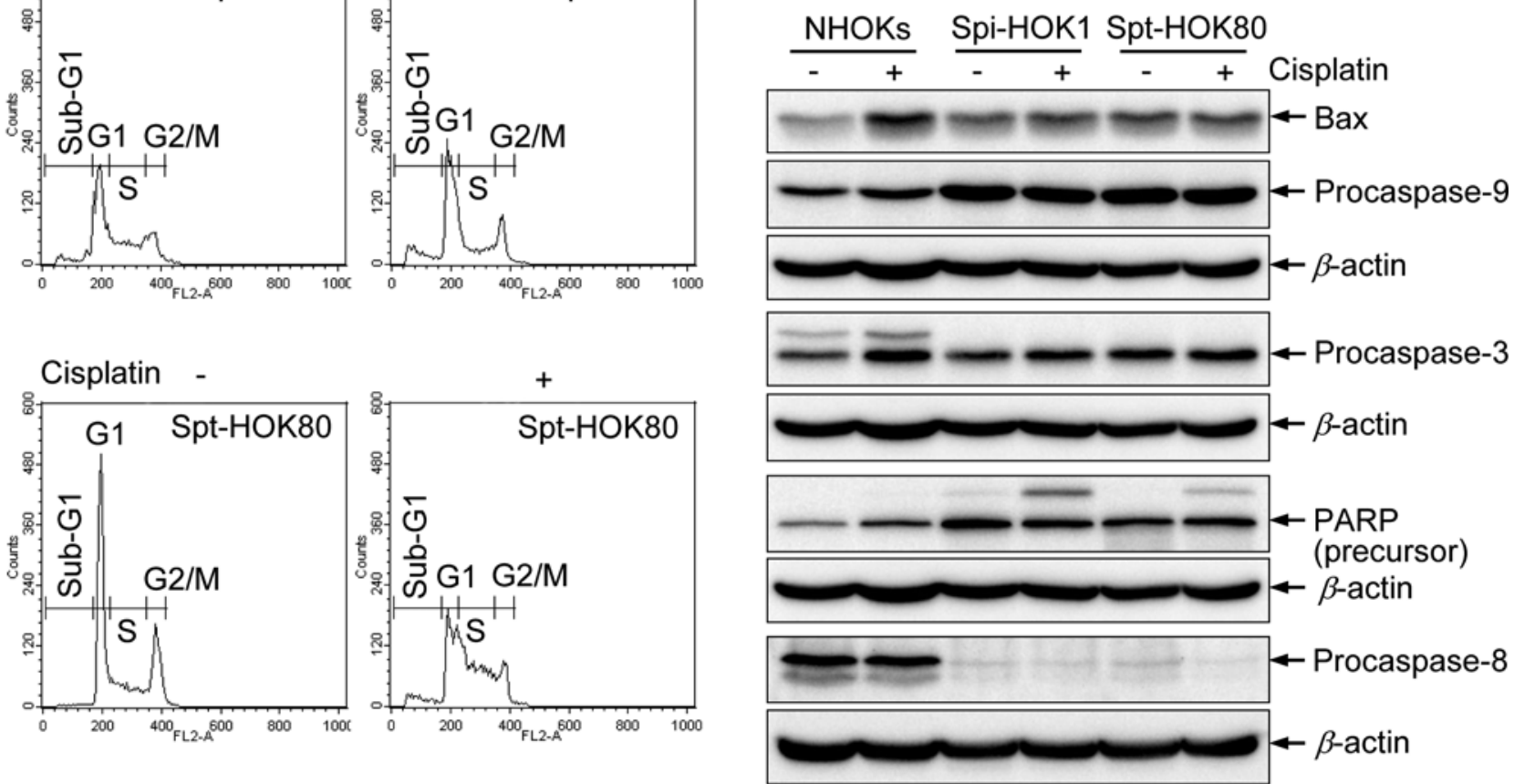

Figure 6. Decreased sensitivity to apoptosis in two spontaneously transformed cell lines. (A) Cell cycle profile analysis. NHOKs, Spi-HOK1 and Spt-HOK80 cells were treated with $25 \mu \mathrm{M}$ cisplatin for $24 \mathrm{~h}$ and submitted to flow cytometry analyses. Data are representative of three separate experiments with identical pattern. Histogram reporting the mean value of the percentage of sub-G1 cell population obtained in three independent experiments. Significant difference versus NHOKs; ${ }^{*} \mathrm{P}<0.05$. (B) Western blot analysis of apoptosis-related proteins in NHOKs, Spi-HOK1 and Spt-HOK80 cells cultured for $24 \mathrm{~h}$ in the presence of $25 \mu \mathrm{M}$ cisplatin.

however, Spi-HOK1 and Spt-HOK80 cell lines showed significant suppression of cisplatin-induced apoptosis relative to NHOKs control (Fig. 6A). This result demonstrates that spontaneously transformed human oral keratinocytes are resistant to cisplatin-induced apoptosis, with the $\mathrm{p} 53^{-/-}$cells being more resistant than $\mathrm{p} 53^{+/+}$cells. An explanation of how spontaneously transformed human oral keratinocytes cause suppression of apoptosis was sought by evaluating the levels of apoptosis-related protein expression in NHOKs, Spi-HOK1 and Spt-HOK80 cells cultured for $24 \mathrm{~h}$ in the presence of $25 \mu \mathrm{M}$ cisplatin. The level of Bax was notably increased in NHOKs exposed to cisplatin but such changes were not observed from the spontaneously transformed Spi-HOK1 and Spt-HOK80 cells in the same conditions (Fig. 6B). Similarly, the levels of procaspase-9, procaspase- 3 and PARP were considerably increased in NHOKs exposed to cisplatin (Fig. 6B). Such changes, however, were not observed in Spi-
HOK1 and Spt-HOK80 cells in the same conditions. The levels of procaspase- 8 in NHOKs, Spi-HOK1 and Spt-HOK80 cells were retained the same in the absence and presence of cisplatin. Taken together, these results demonstrate that spontaneously transformed Spi-HOK1 and Spt-HOK80 lines are resistant to cisplatin-induced apoptosis by suppressing induction of apoptotic proteins, when compared to NHOKs under same conditions.

\section{Discussion}

Although spontaneous immortalization of human epidermal keratinocytes in vitro has been reported previously (21-23), spontaneous immortalization and further spontaneous tumorigenic transformation of human oral keratinocytes in vitro has not yet been reported. The present study demonstrates the spontaneous immortalization and tumorigenic transformation 
of human oral keratinocytes from histologically normal adult gingivas. The Spi-HOK1 line is immortalized, but nontumorigenic in vivo; however, the Spt-HOK80 line is tumorigenic and invasive in vivo. The Spi-HOK1 line is clonal in origin as indicated by a specific and stable cytogenetic marker. This clearly demonstrates that spontaneous immortalization and tumorigenic transformation of human oral keratinocytes in vitro can occur, although cases have not been reported.

Etiological factors for oral cancers are well-established (2); however, a proper in vitro model for studying human oral carcinogenesis has not been previously established. Furthermore, despite advances in cancer diagnosis and treatment, there has been little improvement in the five-year survival rate of oral cancer patients over the last few decades. Epidemiological studies have shown that a significant percentage $(\sim 40 \%)$ of oral and head and neck cancers, as well as anogenital cancer lesions, contain high-risk HPVs (3). Therefore, most in vitro studies of human oral carcinogenesis have been performed with oral keratinocytes immortalized by HPV DNA $(7,8,18)$. More than $60 \%$ of oral cancers are not associated with HPV infection, however, establishment of a novel in vitro multistep model using human oral keratinocyte lines without HPV infection is needed for studying the mechanism of oral carcinogenesis and cancer biology. Carcinogenesis is a multistep process that includes initiation, promotion and progression, and it progresses with sequential escape from tumor-suppressive mechanisms, including senescence and crisis. In this study, we isolated and characterized both spontaneously immortalized and spontaneously tumorigenic-transformed human oral keratinocytes without HPV infection. Because it is thought that spontaneous transformation does not occur in human cells, it was critical to prove the identity of the transformed cells with the original donors. We confirmed the human origins of Spi-HOK1 and Spt-HOK80 lines by karyotyping and demonstrated that these cell lines both had human male karyotypes. Each of the transformed cell lines exhibited a specific abnormal karyotype. The cytogenetic data also documented individual marker chromosomes, which virtually excludes the possibility of cross-contamination. To confirm that the transformed cell lines are epithelial in origin, we examined the expression of cytokeratin and involucrin in both cell lines by immunoperoxidase staining and RT-PCR analysis, respectively, and the cell lines showed epithelial originality with positive results for the expression of both cytokeratin and involucrin.

The mechanism by which oral cancer develops, particularly spontaneous tumorigenicity, has remained largely unknown. The relatively low success rate for transformation of human cells in vitro has mainly been attributed to the higher genetic stability of human cells as compared with rodent cells $(14,16)$. Elucidation of the mechanism that contributes to the induction of oral cancers without HPV infection is of major importance. Here, we found that spontaneously transformed Spi-HOK1 and Spt-HOK80 lines have numerical and structural chromosomal abnormalities and that they have gained and lost entire chromosomes or regions of chromosomes, which is a common characteristic of solid tumors (35). These findings suggest that polyploidization is crucial for autonomous growth in vitro. This conclusion is partly supported by a previous report (21), which explains that polyploidization, which further contributes to genomic imbalance and altered gene expression, may be crucial for the acquisition of autonomous growth in culture but is certainly not sufficient for malignant conversion. Because the p53-mediated cellular responses to stress include cell cycle arrest and apoptosis, and wild-type p53 has also been implicated in cellular senescence (22), we investigated the p53 status of the Spi-HOK1 and Spt-HOK80 lines. In this study, we demonstrated the absence of HPV infection, but the presence of a p53 mutation in the Spi-HOK1 and SptHOK80 lines. This finding suggests that a spontaneous transformation of human oral keratinocytes can occur without HPV infection and that the growth advantage of these lines had been provided by the inactivation of $\mathrm{p} 53$. As the $\mathrm{p} 53$ and $\mathrm{Rb}$ pathways are central in the control of cellular proliferation and senescence, it is not surprising that alterations within these pathways are frequently detected in cancers and transformed cell lines. In fact, mutations in the $p 53$ gene are the most common genetic alteration in a variety of human tumors, including OSCCs and cell lines $(29,32)$. Among these mutations, the missense mutation at codon 175 (CGC to CTC) has been reported in various human cancers, including lung, breast, colon, esophagus and liver (p53 mutation data base; p53.genome.ad.jp). The $p 53$ gene contains a strong transcriptional activation sequence, and p53-induced genes mediate the biological effects of $p 53$ as a tumor suppressor (36). A $21 \mathrm{kDa}$ tumor suppressor protein, $\mathrm{p} 21^{\mathrm{WAF} 1 / \mathrm{Cip} 1}$ is the product of one of the p53-induced genes. Moreover, p2 $1^{\mathrm{WAF} 1 / \mathrm{Cip} 1}$ is a potent inhibitor of cyclindependent kinases, which can suppress tumor cell growth $(33,37)$. In this study, we revealed silencing of $\mathrm{p} 21^{\mathrm{WAF} 1 / \mathrm{Cip} 1}$ expression in the Spi-HOK1 and Spt-HOK80 cells, which have a missense mutation at codon 175 of $p 53$. Furthermore, both cell lines showed increased expression of $\mathrm{p}-\mathrm{Rb}$ proteins. In particular, the levels of p-Rb-Ser807/811 were steadily increased during the process of increased tumorigenicity of human oral keratinocytes. Phosphorylation of $\mathrm{Rb}$ has been known to prevent its association with E2Fs, thereby permitting E2Fs to activate transcription of genes required for cell proliferation. This suggests that increased level of p$\mathrm{Rb}-$ Ser807/811 might be involved in spontaneous tumorigenicity of human oral keratinocytes.

The relationship between spontaneous tumorigenicity of human oral keratinocytes and impaired apoptosis of the cells has not been reported. Currently, one mechanism of action for tumorigenicity is inhibition of apoptosis. Apoptosis is critical for maintaining tissue homeostasis, and impaired apoptosis is now recognized to be a key step in tumorigenesis (34). Here we found that spontaneously transformed SpiHOK1 and Spt-HOK80 lines are resistant to cisplatininduced apoptosis by suppressing induction of apoptotic proteins, when compared to NHOKs. Whether a cell should live or die is largely determined by the Bcl-2 family of antiand proapoptotic regulators (34). The upregulation of Bcl-2 protects cells from apoptosis, whereas cells with increased Bax expression undergo apoptosis by suppressing Bcl-2 activity (38). In this study, increased Bax expression was observed in $\mathrm{NHOKs}$, with the $\mathrm{p} 53^{+/+}$cells, exposed to cisplatin; however, such changes were not observed from the 
spontaneously transformed Spi-HOK1 and Spt-HOK80 cells, with the $553^{--}$cells. The expression of Bax is regulated by the tumor suppressor p53 and has been shown to be involved in p53-mediated apoptosis. Cytochrome $\mathrm{c}$ is involved in chemically-induced apoptotic signaling, which results in the activation of caspases, including caspase-3, leading to PARP degradation (39). In this study, the levels of procaspase-9, procaspase-3 and apoptosis-linked protease PARP in NHOKs exposed to cisplatin was increased, but not in Spi-HOK1 and Spt-HOK80 cells in the same conditions, suggesting that spontaneously transformed Spi-HOK1 and Spt-HOK80 lines were resistant to cisplatin-induced apoptosis by suppressing induction of apoptotic proteins.

In summary, our results constitute the first report to demonstrate that Spi-HOK1 and Spt-HOK80 are novel cell lines that are spontaneously immortalized and tumorigenictransformed from human oral keratinocytes without HPV infection, respectively. Thus, these spontaneously transformed cell lines may be useful in various experimental models for investigation of in vitro multistep oral carcinogenesis and oral biology.

\section{Acknowledgments}

This work was supported by a grant (Grant No. 2007-0052487) from the Korean Science and Engineering Foundation (to B.-M. M.).

\section{References}

1. Parkin D, Bray F, Ferlay J and Pisani P: Global cancer statistics. CA Cancer J Clin 55: 74-108, 2005.

2. McDowell JD: An overview of epidemiology and common risk factors for oral squamous cell carcinoma. Otolaryngol Clin $\mathrm{N}$ Am 39: 229-402, 2006.

3. Forastiere A, Koch W, Trotti A and Sidransky D: Head and neck cancer. N Engl J Med 345: 1890-1900, 2001

4. Regezi JA and Sciubba J: Ulcerative conditions. In: Oral Pathology: Clinical-Pathologic Correlations. Regezi JA and Sciubba J (eds). W.B. Saunders, Philadelphia, pp77-90, 1993.

5. Mizobuchi S, Furihata M, Sonobe H, Ohtsuki Y, Ishikawa T, Murakami H, Kurabayashi A, Ogoshi S and Sasaguri S: Association between p53 immunostaining and cigarette smoking in squamous cell carcinoma of the esophagus. Jpn J Clin Oncol 30: 423-428, 2000

6. Gensier HL, Hall JD and Bernstein H: The DNA damage hypothesis of aging: importance of oxidative damage. In: Review of Biological Research in Aging. Rothstein M (ed). Alan R. Liss, New York, pp451-465, 1987.

7. Park N-H, Min B-M, Li S-L, Huang MZ, Cherrick HM and Doniger J: Immortalization of normal human oral keratinocytes with type 16 human papillomavirus. Carcinogenesis 12: $1627-1631,1991$

8. Min B-M, Woo KM, Baek J-H, Lee G and Park N-H: Malignant transformation of HPV-immortalized human oral keratinocytes by chemical carcinogen. Int J Oncol 7: 249-256, 1995.

9. Hayflick L and Moorhead PS: The serial cultivation of human diploid cell strains. Exp Cell Res 25: 585-621, 1961.

10. Min B-M, Woo KM, Lee G and Park N-H: Terminal differentiation of normal human oral keratinocytes is associated with enhanced cellular TGF- $\beta$ and phospholipase C- $\gamma 1$ levels and apoptotic cell death. Exp Cell Res 249: 377-385, 1999.

11. Kang MK, Guo W and Park N-H: Replicative senescence of normal human oral keratinocytes is associated with the loss of telomerase activity without shortening of telomeres. Cell Growth Differ 9: 85-95, 1998

12. Maruoka Y, Harada H, Mitsuyasu T, Seta Y, Kurokawa H, Kajiyama $\mathrm{M}$ and Toyoshima K: Keratinocytes become terminally differentiated in a process involving programmed cell death. Biochem Biophys Res Commun 238: 886-890, 1997.
13. Sanford KK and Evans VJ: A quest for the mechanism of 'spontaneous' malignant transformation in culture with associated advances in culture technology. J Natl Cancer Inst 68: 895-913, 1982.

14. DiPaolo JA: Relative difficulties in transforming human and animal cells in vitro. J Natl Cancer Inst 70: 3-8, 1983.

15. Barrett JC and Tennant RW: Mammalian cell transformation: mechanisms of carcinogenesis and assays for carcinogens. In: Carcinogenesis: A Comprehensive Survey. Barrett JC and Tennant RW (eds). Raven Press, New York, pp90-117, 1985.

16. Sager R, Tanaka K, Lau CC, Ebina Y and Anisowicz A: Resistance of human cells to tumorigenesis induced by cloned transforming genes. Proc Natl Acad Sci USA 80: 7601-7605, 1983.

17. Kano Y and Little JB: Mechanisms of human cell neoplastic transformation: X-ray induced abnormal clone formation in long-term cultures of human diploid fibroblasts. Cancer Res 45 : 2550-2555, 1985.

18. Shin K-H, Min B-M, Cherrick HM and Park N-H: Combined effects of human papillomavirus-18 and $N$-Methyl-N'-nitroNnitrosoguanidine on the transformation of normal human oral keratinocytes. Mol Carcinog 9: 76-86, 1994.

19. Kubo C, Tsutsui TW, Tamura Y, Kumakura S and Tsutsui T: Immortalization of normal human gingival keratinocytes and cytological and cytogenetic characterization of the cells. Odontology 97: 18-31, 2009.

20. Lee KM, Choi KH and Ouellette MM: Use of exogenous hTERT to immortalize primary human cells. Cytotechnology 45: 33-38, 2004.

21. Boukamp P, Petrussevska RT, Breitkreutz D, Hornung J, Markham A and Fusenig NE: Normal keratinization in a spontaneously immortalized aneuploid human keratinocyte cell line. J Cell Biol 106: 761-771, 1988.

22. Allen-Hoffmann B L, Schlosser SJ, Ivarie CAR, Sattler CA, Meisner LF and O'Connor SL: Normal growth and differentiation in a spontaneously immortalized near-diploid human keratinocyte cell line, NIKS. J Invest Dermatol 114: 444-455, 2000.

23. Rea MA, Zhou L, Qin Q, Barrandon Y, Easley KW, Gungner SF, Phillips MA, Holland WS, Gumerlock PH, Rocke DM and Rice RH: Spontaneous immortalization of human epidermal cells with naturally elevated telomerase. J Invest Dermatol 126: 2507-2515, 2006.

24. Oh J-E, Kook J-K and Min B-M: Big-h3 induces keratinocyte differentiation via modulation of involucrin and transglutaminase expression through the integrin $\alpha 3 \beta 1$ and the phosphatidylinositol 3-kinase/Akt signaling pathway. J Biol Chem 280: 21629-21637, 2005.

25. Olshan AF, Weissler MC, Pei H, Conway K, Anderson S, Fried DB and Yarbrough WG: Alterations of the p16 gene in head and neck cancer: frequency and association with p53, PRAD-1 and HPV. Oncogene 14: 811-818, 1997.

26. Ansari-Lari MA, Staebler A, Zaino RJ, Shah KV and Ronnett BM: Distinction of endocervical and endometrial adenocarcinomas: immunohistochemical p16 expression correlated with human papillomavirus (HPV) DNA detection. Am J Surg Pathol 28: 160-167, 2004.

27. Slebos RJC, Yi Y, Ely K, Carter J, Evjen A, Zhang X, Shyr Y, Murphy BM, Cmelak AJ, Burkey BB, Netterville JL, Levy S, Yarbrough WG and Chung $\mathrm{CH}$ : Gene expression differences associated with human papillomavirus status in head and neck squamous cell carcinoma. Clin Cancer Res 12: 701-709, 2006.

28. Fregonesi PAG, Teresa DB, Duarte RA, Neto CB, Oliveira MRD and Soares CP: p16 $6^{\mathrm{INK} 4 \mathrm{~A}}$ immunohistochemical overexpression in premalignant and malignant oral lesions infected with human papillomavirus. J Histochem Cytochem 51: 1291-1297, 2003.

29. Ries JC, Schreiner D, Steininger H and Girod SC: p53 mutation and detection of p53 protein expression in oral leukoplakia and oral squamous cell carcinoma. Anticancer Res 18: 2031-2036, 1998.

30. van Heerden WF, van Rensburg EJ, Hemmer J, Raubenheimer EJ and Engelbrecht S: Correlation between p53 gene mutation, p53 protein labeling and PCNA expression in oral squamous cell carcinomas. Anticancer Res 18: 237-240, 1998.

31. Min B-M, Baek J-H, Shin K-H, Gujuluva CN, Cherrick HM and Park N-H: Inactivation of the p53 gene by either mutation or HPV infection is extremely frequent in human oral squamous cell carcinoma cell lines. Eur J Cancer B Oral Oncol 30B: 338-345, 1994.

32. Lane DP: On the expression of the $\mathrm{p} 53$ protein in human cancer. Mol Biol Rep 19: 23-29, 1994. 
33. El-Deiry WS, Tokino T, Velculescu VE, Levy DB, Parsons R, Trent JM, Lin D, Mercer WE, Kinzler KW and Vogelstein B: WAF1, a potential mediator of p53 tumor suppression. Cell 75: $817-825,1993$

34. Cory S, Huang David CS and Adams JM: The Bcl-2 family: roles in cell survival and oncogenesis. Oncogene 22: 85908607,2002

35. Kops GJ, Weaver BA and Cleveland DW: On the road to cancer: aneuploidy and the mitotic checkpoint. Nat Rev Cancer 5: 773-785, 2005.

36. Vogelstein B and Kinzler KW: p53 function and dysfunction. Cell 70: 523-526, 1992
37. Harper JW, Adami GR, Wei N, Keyomarsi K and Elledge SJ: The p21 Cdk-interacting protein Cip1 is a potent inhibitor of G1 cyclin-dependent kinases. Cell 75: 805-816, 1993.

38. Reed JC: Dysregulation of apoptosis in cancer. J Clin Oncol 17: 2941-2953, 1999.

39. Sun XM, MacFarlane M, Zhuang J, Wolf BB, Green DR and Cohen GM: Distinct caspase cascades are initiated in receptormediated and chemical-induced apoptosis. J Biol Chem 274: 5053-5060, 1999. 